\title{
Caracterização de nascidos vivos prematuros em um município do nordeste brasileiro
}

Characterization of preterm live births in a municipality in northeastern Brazil

Caracterización de nacidos vivos prematuros en un municipio del noreste de Brasil

Luciano Marques dos Santos ${ }^{1}$ (D) https://orcid.org/0000-0001-7866-6353

Taynara Bispo Conceição ${ }^{1}$ (D) https://orcid.org/0000-0002-6453-762X

Aline Silva Gomes ${ }^{1}$ (D) https://orcid.org/0000-0002-3012-6849

Cleonara Sousa Gomes e Silva ${ }^{1}$ (D) https://orcid.org/0000-0002-4827-8306

Michele de Santana Xavier Ramos ${ }^{1}$ (D) https://orcid.org/ 0000-0001-6679-207X

Silvia da Silva Santos Passos ${ }^{1}$ (D) https://orcid.org/0000-0002-2104-5131

Silvone Santa Bárbara da Silva Santos ${ }^{1}$ (D) https://orcid.org/0000-0001-5681-7894

\section{Resumo}

Objetivo: Caracterizar os nascidos vivos prematuros em um município do nordeste brasileiro quanto às características demográficas, clínicas, necessidade de reanimação e hospitalização.

Métodos: Estudo descritivo, realizado em dois hospitais da Bahia, com a participação de 333 nascidos vivos prematuros. Os dados foram coletados no período de abril de 2014 a fevereiro de 2016, tabulados e analisados SPSS versão 22.0. Foi aprovado por Comitê de Ética.

Resultados: os nascidos vivos prematuros foram em sua maioria do sexo masculino, com baixo peso ao nascer, Apgar média de 7 no primeiro e 8 no quinto minuto de vida. A maioria foi classificado como prematuro tardio por meio da DUM, ecografia e método de Capurro. Referente a assistência imediata no nascimento, 60,5\% dos recém-nascidos foram reanimados.

Conclusão: Apontam a necessidade de capacitação dos trabalhadores da saúde em cuidados clínicos avançados e o planejamento de medidas eficazes que visem reduzir complicações ao nascimento.

\section{ABSTRACT}

Objective: To characterize premature live births in a municipality in northeastern Brazil regarding demographic and clinical characteristics, the need for resuscitation and hospitalization.

Methods: Descriptive study, carried out in two hospitals in Bahia, with the participation of 333 premature live births. Data were collected from April 2014 to February 2016, tabulated and analyzed SPSS version 22.0. It was approved by the Ethics Committee.

Results: Preterm live births were mostly male, with low birth weight, mean Apgar score of 7 in the first and 8 in the fifth minute of life. Most were classified as late preterm by means of LMP, ultrasound and Capurro method. Regarding immediate assistance at birth, $60.5 \%$ of newborns were resuscitated, and $21 \%$ received inhaled oxygen. Conclusion: They point out the need for training health workers in advanced clinical care and the use of low-cost strategies, as well as planning effective measures to reduce complications at birth.

\section{Resumen}

Objetivo: Caracterizar los nacimientos vivos prematuros en un municipio del noreste de Brasil con respecto a las características demográficas y clínicas, la necesidad de reanimación y hospitalización.

Métodos: Estudio descriptivo, realizado en dos hospitales de Bahía, con la participación de 333 nacimientos prematuros vivos. Los datos se recopilaron de abril de 2014 a febrero de 2016, se tabularon y analizaron SPSS versión 22.0. Fue aprobado por el Comité de Ética.

Resultados: Los nacimientos vivos prematuros fueron en su mayoría varones, con bajo peso al nacer, puntaje promedio de Apgar de 7 en el primer minuto y 8 en el quinto minuto de vida. La mayoría fueron clasificados como prematuros tardíos mediante LMP, ultrasonido y método Capurro. Con respecto a la asistencia inmediata al nacer, el $60.5 \%$ de los recién nacidos fueron resucitados y el $21 \%$ recibió oxígeno inhalado.

Conclusion: Señalan la necesidad de capacitar a los trabajadores de la salud en atención clínica avanzada y el uso de estrategias de bajo costo, así como planificar medidas efectivas para reducir las complicaciones al nacer.

\section{Como citar:}

Santos LM, Conceição TB, Gomes AS, Gomes e Silva CS, Ramos MS, Passos SS, et al. Caracterização de nascidos vivos prematuros em um município do nordeste brasileiro. Rev Soc Bras Enferm Ped. 2021;21(2):85-90.

\footnotetext{
${ }^{1}$ Universidade Estadual de Feira de Santana, Feira de Santana, BA, Brasil.

Conflitos de interesse: nada a declarar.

Submetido: 26 de Abril de 2021 | Aceito: 3 de Dezembro de 2021

Autor correspondente: Luciano Marques dos Santos | E-mail: lucmarxenfo@yahoo.com.br

DOI: http://dx.doi.org/10.31508/1676-379320210013
}

\section{Keywords}

Obstetric nursing; Obstetric labor, Premature; Infant preterm; Pediatric nursing

\section{Descriptores}

Enfermería obstétrica; Trabajo de parto prematuro; Recien nacido prematuro; Enfermería pediátrica 


\section{Introdução}

O recém-nascido é considerado prematuro quando nasce com menos de 37 semanas completas (259 dias), contadas a partir do primeiro dia do último período menstrual. ${ }^{(1)}$ Apesar dos avanços tecnológicos e desenvolvimento do país, as taxas de prematuridade permanecem elevadas. ${ }^{(2)}$ A taxa global estimada de nascimentos prematuros para 2014 foi $10,6 \%$, sendo que para o Brasil esta estimativa foi bem maior $(11,6 \%)$. $^{(3)}$

A prematuridade é uma questão de saúde pública, por ser um fator determinante da morbimortalide neonatal. ${ }^{(4)}$ Com o nascimento de uma criança prematura, faz-se necessário um local que possua recursos tecnológicos, humanos e terapêuticos especializados a fim de proporciona-lo cuidados complexos. Esses suportes são encontrados nas Unidades de Terapia Intensiva, onde a assistência ocorre de maneira continua, e cujo surgimento tem proporcionando um aumento na sobrevida orgânica dos prematuros, principalmente os extremos e de baixo peso. ${ }^{(1)}$

A identificação do perfil demográfico e clínico das crianças que nascem prematuramente em um município do nordeste brasileiro torna-se relevante, uma vez que tal conhecimento permitirá uma descrição dos recém-nascidos assistidos, norteando quais intervenções terapêuticas necessitam ser tomadas para controlar as condições clínicas e determinação social desse agravo. Ademais, a avaliação contínua dos indicadores de saúde dessa população, poderá trazer informações necessárias para o planejamento de estratégias de promoção da assistência integral à criança e redução da morbimortalidade infantil.

Desta forma, questiona-se: Quais as características demográficas, clínicas, necessidade de reanimação e hospitalização dos nascidos vivos prematuros em um município do nordeste brasileiro? O objetivo foi caracterizar os nascidos vivos prematuros em um município do nordeste brasileiro quanto às características demográficas, clínicas, necessidade de reanimação e hospitalização.

\section{Métodos}

Trata-se de um estudo descritivo, com os dados extraídos de um dos centros nordestino de uma pes- quisa multicêntrica nacional do tipo caso-controle. Foi realizada em dois hospitais do nordeste brasileiro, no estado da Bahia. Para elaboração deste relato de pesquisa utilizou-se o instrumento Strengthening the Reporting of Observational Studies in Epidemiology (STROBE).

A amostra da pesquisa multicêntrica foi estimada em 600 puérperas/recém-nascidos, divididos em 300 casos e 300 casos controles, tomando como base dados do município do Rio Grande, e pelo fato do Rio Grande do Sul ter o maior índice de prematuridade entre os estados brasileiros. Este cálculo foi realizado esperando uma prevalência de partos prematuros em torno de $12 \%$, com o máximo de $10 \%$ de perdas. Para esta pesquisa, foram utilizados os dados dos 333 casos, em particular, dos recém-nascidos prematuros. Portanto, não foram adotados critérios de inclusão e de exclusão.

Foi considerado prematuro o recém-nascido com idade gestacional menor do que 37 semanas, contadas a partir do primeiro dia da data da última menstruação (DUM), ecografia e método de Capurro. A prematuridade foi classificada conforme o número de semanas de idade gestacional em tardia, entre 34 e 36 semanas; moderada, entre 32 e 33 semanas; muito prematuro, entre 28 e 31 semanas e o prematuro extremo menos de 28 semanas. ${ }^{(5)}$

A coleta de dados ocorreu entre abril de 2014 a fevereiro de 2016 por seis pesquisadoras devidamente qualificadas para a aplicação do formulário que continha dados maternos e do recém-nascido. Foram coletadas informações de dados primários por meio de entrevistas com os puérperas, e os dados secundários foram obtidos em prontuários, sendo observada as seguintes variáveis: sexo, idade gestacional, peso ao nascer, Apgar no primeiro e quinto minuto de vida, necessidade de reanimação, medidas utilizadas durante a reanimação, necessidade de hospitalização, local e motivos da hospitalização.

Os dados foram digitados duplamente no Software Package for Social Sciences (SPSS), versão 22.0 e apresentados por meio de suas frequências absolutas e relativas. As variáveis quantitativas foram sumarizadas por meio de medidas de tendência central e dispersão. Esta pesquisa foi aprovada por Comitê de Ética, parecer de número 134/2013. 


\section{Resultados}

Foram observadas $51,1 \%$ das crianças classificadas como prematuras de um total de 653 recém-nascidos hospitalizados. Identificado à caracterização dos nascidos vivos prematuros, necessidade de reanimação em sala de parto e cuidados intensivos ou semi-intensivos. De acordo com as variáveis relacionadas ao recém-nascido prematuro, a maioria foi do sexo masculino, com baixo peso ao nascer, Apgar maior do que 7 no primeiro e quinto minuto de vida. A idade gestacional foi classificada como prematuro tardio, tanto por meio da DUM, ecografia e método de Capurro (Tabela 1).

No que se refere a assistência imediata no nascimento (Tabela 1), a maioria dos recém-nascidos foram reanimados, com destaque para o uso de oxigênio inalatório, e encaminhados para unidade de alojamento conjunto ou unidade semi-intensiva neonatal. Nesta última unidade, o motivo da hospitalização foi a disfunção respiratória.

\section{Discussão}

Quanto às características dos prematuros nascidos vivos, esta pesquisa mostrou predomínio de recém-nascidos do sexo masculino, semelhante a dados de outras pesquisas. ${ }^{(6-8)} \mathrm{Na}$ presente pesquisa, $74.9 \%$ dos recém-nascidos prematuros apresentaram baixo peso ao nascer, o que foi observado em pesquisas anteriores. ${ }^{(9,10)}$ Prematuros com peso inferior a $1.500 \mathrm{Kg}$ permanecem por mais de 30 dias hospitalizados em Unidade de Terapia Intensiva Neonatal. ${ }^{(11)}$

O peso ao nascer inferior a $2.500 \mathrm{~g}$ é considerado um dos principais determinantes da mortalidade neonatal e da morbidade infantil, sendo fator decisivo para a análise do desenvolvimento pós-natal. ${ }^{(9)}$ Em um pesquisa internacional, na análise multivariada, verificou-se que recém-nascidos com peso inferior a 2,500kg tiveram maior prevalência de asfixia perinatal. ${ }^{(12)}$

Coorte retrospectiva realizada no município do Rio de Janeiro, observou que a maior probabilidades de morte foi encontrada entre nascidos vivos do sexo masculino, com peso ao nascer inferior a $2.500 \mathrm{~g}$ e prematuros. Os autores destacaram que a promoção de cuidados perinatais pelos serviços de saúde poderia evitar cerca de $65,0 \%$ desses óbitos. ${ }^{(13)}$
Tabela 1. Características de nascidos vivos prematuros e assistência imediata em dois hospitais públicos de um município do nordeste brasileiro

\begin{tabular}{|c|c|c|c|}
\hline Variáveis & $\mathrm{n}(\%)$ & Média & $\begin{array}{l}\text { Desvio } \\
\text { Padrão }\end{array}$ \\
\hline \multicolumn{4}{|l|}{ Sexo $(n=333)$} \\
\hline Masculino & $168(50,6)$ & & \\
\hline Feminino & $164(49,4)$ & & \\
\hline \multicolumn{4}{|l|}{ Peso ao Nascer $\left(n=331^{*}\right)$} \\
\hline Baixo peso extremo & $19(5,7)$ & 2.091 & $690 \mathrm{~g}$ \\
\hline Muito baixo peso & $46(13,9)$ & & \\
\hline Baixo peso ao nascer & $183(55,3)$ & & \\
\hline Peso normal & $83(25,1)$ & & \\
\hline \multicolumn{4}{|l|}{ Apgar $1(n=301 *)$} \\
\hline$<7$ & $67(22,3)$ & 7 & 1,644 \\
\hline$\geq 7$ & $234(77,7)$ & & \\
\hline \multicolumn{4}{|l|}{ Apgar $5(n=301 *)$} \\
\hline$<7$ & $21(7,0)$ & 8 & 1,258 \\
\hline$\geq 7$ & $280(93,0)$ & & \\
\hline \multicolumn{4}{|l|}{$\operatorname{DUM}\left(n=231^{*}\right)$} \\
\hline Prematuro extremo & $15(6,5)$ & 34,3 & \\
\hline Muito prematuro & $37(16)$ & & \\
\hline Prematuro moderado & $30(13)$ & & \\
\hline Prematuro tardio & $149(64,5)$ & & \\
\hline \multicolumn{4}{|l|}{ Ecografia $(n=300 *)$} \\
\hline Prematuro extremo & $14(4,7)$ & 34 & \\
\hline Muito prematuro & $46(15,3)$ & & \\
\hline Prematuro moderado & $65(21,7)$ & & \\
\hline Prematuro tardio & $175(58,3)$ & & \\
\hline \multicolumn{4}{|l|}{ Método de Capurro ( $\mathrm{n}=219 *$ ) } \\
\hline Prematuro extremo & $6(2,8)$ & 34 & \\
\hline Muito prematuro & $48(21,9)$ & & \\
\hline Prematuro moderado & $48(21,9)$ & & \\
\hline Prematuro tardio & $117(53,4)$ & & \\
\hline \multicolumn{4}{|c|}{$\begin{array}{l}\text { Necessidade de alguma medida de } \\
\text { reanimação }\left(n=332^{*}\right)\end{array}$} \\
\hline $\operatorname{Sim}$ & $201(60,5 \%)$ & & \\
\hline Não & $131(39,5 \%)$ & & \\
\hline \multicolumn{4}{|c|}{$\begin{array}{l}\text { Que atendimento necessitou em sala } \\
\text { de parto }\left(n=175^{*}\right)\end{array}$} \\
\hline Oxigênio inalatório & $70(21,0)$ & & \\
\hline VPP por máscara & $25(7,5)$ & & \\
\hline VPP por TET & $20(6,0)$ & & \\
\hline Massagem cardíaca externa & $4(1,2)$ & & \\
\hline Medicamentos & $4(1,2)$ & & \\
\hline Outro & $52(15,7)$ & & \\
\hline \multicolumn{4}{|l|}{ Local da hospitalização ( $n=333$ ) } \\
\hline Alojamento conjunto & $141(42,3)$ & & \\
\hline Semi-Intensiva Neonatal & $107(32,2)$ & & \\
\hline $\begin{array}{l}\text { Unidade de Terapia Intensiva } \\
\text { Neonatal }\end{array}$ & $85(25,5)$ & & \\
\hline \multicolumn{4}{|l|}{$\begin{array}{l}\text { Motivo principal da internação } \\
\left(\mathrm{n}=195^{*}\right)\end{array}$} \\
\hline Disfunção respiratória & $137(41,2)$ & & \\
\hline Outros & $58(17,6)$ & & \\
\hline
\end{tabular}

*As diferenças no total da amostra são decorrentes de dados ignorados que não foram encontrados no prontuário do recém-nascido ou materno, ou a entrevistada não soube/quis informar, ou não se aplicam a questão. 
Os recém-nascidos da presente pesquisa foram classificados em sua maioria como prematuros tardios, o que está de acordo com pesquisa mundial que destacou esta classificação em quase $85 \%$ dos nascimentos prematuros ${ }^{(5)}$ e outra nacional. ${ }^{(14)}$

$\mathrm{Na}$ atual pesquisa, a idade gestacional foi determinada por meio da DUM, ecografia e método de Capurro. No entanto, este método não é o mais indicado para este tipo de avaliação em recém-nascidos prematuros, pois enfatiza mais características somáticas em detrimento das neurológicas. Para tanto, em crianças prematuras o cálculo da idade gestacional deve ser utilizado o Escore de New Ballard por considerar diversas características neurológicas e somáticas. Entretanto, nas unidades pesquisadas o referido escore não é utilizado na avaliação da idade gestacional do recém-nascido prematuro.

Uma avaliação precisa da idade gestacional é primordial para tomar decisões sobre cuidados obstétricos que afetem o recém-nascido prematuro. Para obter a idade gestacional precisa e confiável em alguns casos, torna-se difícil devido a fatores, como a incerteza quanto à data da DUM e o início tardio do pré-natal, condições que alteram a idade gestacional baseada no cálculo pelo ultrassom. ${ }^{(15)}$

A maioria dos recém-nascidos da presente pesquisa apresentaram escore de Apgar maior ou igual a 7 pontos tanto no primeiro, quanto no quinto minuto de vida, concordando com pesquisa nacional. ${ }^{(16)}$ No entanto, no primeiro minuto de vida, $22,3 \%$ este valor foi menor que 7 e $7 \%$ obtiveram este valor no quinto minuto de vida. Entretanto, recém-nascido com índice de Apgar no $5^{\circ}$ minuto de zero a três pontos apresentaram 4 vezes mais chances de prematuridade. ${ }^{(10)}$

$O$ índice de Apgar menor que 7 identifica os recém-nascidos que necessitam de cuidados intervenções imediatas pela equipe de saúde atuante em salas de parto, o que não indica necessariamente em alguma manobra de reanimação, mas que o recém-nascido não apresentou adequada transição da vida fetal para a extrauterina.

Na presente pesquisa, $60,5 \%$ dos recém-nascidos necessitaram de reanimação em sala de parto. Com relação a necessidade de reanimação em prematuros, a idade gestacional e peso ao nascer influenciam nas condições dos recém-nascidos e na necessidade desta intervenção, sendo a mortalidade maior quanto a idade gestacional e/ou peso ao nascer forem menores. ${ }^{(6)}$
Durante a reanimação, 21\% dos recém-nascidos prematuros receberam oxigênio inalatório, sendo baixo os percentuais de utilização da ventilação com pressão positiva por máscara, intubação traqueal, massagem cardíaca externa e administração de medicamentos. Porém, pesquisa realizada nos Estados Unidos da América apontou que as taxas de intubação aumentaram para 68\% em recém-nascidos com 23 semanas e 87\% em 24 semanas, diminuindo após a $24^{\underline{a}}$ semanas de idade gestacional. De 856 recém-nascidos prematuros que receberam medicamentos e/ou compressões torácicas, 96\% também foram submetidos à intubação. ${ }^{(17)}$

Dados de pesquisa retrospectiva realizado em Minas Gerais demonstrou que recém-nascidos com baixo escore de Apgar menor ou igual a 3 no foram submetidos aos passos iniciais da reanimação neonatal e 64,3\% presentaram recuperação no Apgar de 5ำ minuto de vida. Dentre aqueles que receberam alguma intervenção, 63,8\% recuperou com ventilação com máscara, sendo que houve menor taxa de recuperação em RN com idade gestacional $<34$ semanas. ${ }^{(18)}$

A respiração imediatamente após o nascimento iniciada por meio de estimulação em cerca de $10 \%$ dos recém-nascidos, enquanto que $5 \%$ demandam alguma intervenção mais complexa. Destes últimos, 3\% necessitam de ventilação com pressão positiva e $2 \%$ de intubação orotraqueal para garantir um suporte ventilatório adequado. ${ }^{(19)}$

A ventilação é considerada um ponto crítico para o sucesso da reanimação neonatal, fazendo com que os pulmões do recém-nascido sejam adequadamente inflados e, com assim, ocorra a dilatação da vasos pulmonares e hematose suficiente. ${ }^{(16)}$ Assim, é primordial que as unidades obstétricas possuam equipamentos apropriados e uma equipe qualificada para prontamente iniciar os passos propostos para a reanimação neonatal. ${ }^{(16)}$

Os dados da presente pesquisa sinalizam a necessidade de qualificação dos trabalhadores da saúde envolvidos na atenção ao recém-nascido nos primeiros minutos de vida quanto à adequada reanimação neonatal, pois a oferta de oxigênio inalatório não tem sido mais recomendada no conjunto de intervenções para a resolução da asfixia neonatal devido aos riscos associados.

Dentre os prematuros da pesquisa que necessitaram de atendimento em sala de parto, $42,3 \%$ foram 
hospitalizados em unidade de alojamento conjunto, $32,2 \%$ em unidade semi-intensiva neonatal e $25,5 \%$ em cuidados intensivos. O principal motivo para a hospitalização foi a disfunção respiratória, o que apareceu em outras pesquisas. . $^{(7,17)}$

Quanto às dificuldades adaptativas que recém-nascidos prematuros apresentam, destaca-se suscetibilidade aumentada ao estresse oxidativo e às infecções, o que facilita o aparecimento de morbidades que contribuem para a mortalidade neonatal desse grupo de pacientes. ${ }^{(20)}$

Na primeira hora de vida, recém-nascidos prematuros apresentaram hipotermia associada a fato de serem pequenos para a idade gestacional e necessidade de compressões torácicas em sala de parto. A hiportermia poderá agravar a disfunção respiratória, o que justificará a necessidade do uso da ventilação mecânica.

Neste sentido, o prematuro está exposto à ocorrência de diversos problemas de saúde associados a sua imaturidade anatômica e funcional, com destaque para o sistema imunológico e respiratório, como também, problemas infecciosos, gastrointestinais e ou neurológicos, os quais podem levar a sequelas a médio ou longo prazo.

Para o tratamento dos recém-nascidos prematuros faz-se necessário também a implementação de estratégias de baixo custo, a exemplo da imunoterapia oral com colostro, considerada uma alternativa uma alternativa possível e viável para auxiliar na recuperação da saúde dos neonatos. Recém-nascidos prematuros com muito baixo tem sobrevivido devido ao avanço da neonatologia, no entanto, deve se ter como meta a sobrevivência sem sequelas e com qualidade de vida para esses neonatos e suas famílias.

Dado à metodologia e ao tipo do estudo, destaca-se como limitação a impossibilidade de realizar a inferência dos dados à toda a população. Também, o sub-preenchimento dos prontuários corroborando para falhas na coleta de algumas variáveis.

Para o contexto teórico, a pesquisa possibilitou conhecer o perfil dos prematuros nascidos em uma cidade do interior da Bahia, possibilitando traçar futuras hipóteses sobre a sobrevida e o prognóstico, além de contribuir para o desenvolvimento de pesquisas sobre os fatores que predispõe a ocorrência de prematuridade e a influência das ações assistenciais na recuperação clínica desse público.
Assim, na prática, pode-se pensar em estratégias para melhorar a atenção oferecida à esse público conforme as necessidade do quadro clinico e características apresentadas. Os dados, também, poderão ser utilizados na reorganização da assistência ao recém-nascido prematuro na sala de parto, no contexto estudado.

Enquanto que no meio social, a pesquisa propôs a reflexão sobre a importância de introduzir o recém-nascido no âmbito familiar sem ou com poucas sequelas decorrentes das disfunções provocadas pela prematuridade, mediante o desenvolvimento de esforços dos profissionais em saúde em oferecer uma assistência de qualidade e segura, livre de danos e falhas que repercuta de forma negativa na vida do indivíduo e da sua rede social.

\section{Conclusão}

Na presente pesquisa foram identificadas características clínicas e assistenciais de recém-nascidos prematuros semelhantes a dados de outras nacionais e internacionais. Verificou-se que a maiorias dos recém-nascidos prematuros eram do sexo masculino, baixo peso ao nascer, Apgar maior do que 7 no primeiro e quinto minuto de vida, prematuros tardios, a maioria dos recém-nascidos foram reanimados, usando oxigênio inalatório, e encaminhados para unidade de alojamento conjunto ou unidade semi-intensiva neonatal, sendo o principal motivo da hospitalização a disfunção respiratória. Os resultados apontam para a necessidade da capacitação dos trabalhadores da saúde em cuidados clínicos avançados, como também na utilização de estratégias de baixo custo e planejamento de medidas eficazes que visem reduzir complicações ao nascimento. Sugere-se a necessidade de atenção clínica eficaz para as disfunções respiratórias apresentadas e consequentemente um suporte necessário e ideal aos recém-nascidos.

\section{Agradecimentos}

Ao projeto multicêntrico intitulado "Parto prematuro: estudos dos fatores associados para construção de estratégias de prevenção" (parecer de número 134/2013) 
da Universidade Federal do Rio Grande e ao Programa Estágio Voluntário de Iniciação Científica e Tecnológica (PEVIC) da Universidade Estadual de Feira de Santana.

\section{Contribuições}

Santos LM, Conceição TB, Gomes AS, Gomes e Silva CS, Ramos MSX, Passos SSS e Santos SSBS contribuíram com a concepção do projeto, análise e interpretação dos dados, redação do artigo, revisão crítica relevante do conteúdo intelectual e aprovação da versão final a ser publicada.

\section{Referências}

1. Teixeira $L A$, Vasconcelos $L D$, Ribeiro RA. Prevalência de Patologias e Relação com a Prematuridade em Gestação de Alto Risco. Rev Ciênc Saúde. 2015;5(4):35-42.

2. Santos LM, Ribeiro IS, Santana RC. Identificação e tratamento da dor no recém-nascido prematuro na Unidade de Terapia Intensiva. Rev Bras Enferm. 2012;65(2):269-75.

3. Chawanpaiboon S, Vogel JP, Moller AB, Lumbiganon P, Petzold M, Hogan D. Global, regional, and national estimates of levels of preterm birth in 2014: a systematic review and modelling analysis. Lancet Glob Health. 2019;7:e37-46.

4. World Health Organization (WHO). Survive and thrive: transforming care for every small and sick newborn. Key findings. Geneva: World Health Organization; 2018.

5. American Academy of Pediatrics/AAP \& The American College of Obstetricians and Gynecologists. Guidelines for Perinatal Care Eighth edition. Elk Grove Village, IL, 2017.

6. Drummond S, Souza TS, Lima FG, Vieira AA. Correlação entre 0 uso de corticoterapia antenatal, a reanimação e a mortalidade de recém-nascidos prematuros de muito baixo peso. Rev Bras Ginecol Obstet. 2014 ;36(5):211-5.
7. Cunha AA, Fernandes DS, Melo PF, Guedes MH. Fatores associados à asfixia perinatal. Rev Bras Ginecol Obstet. 2004;26(10):799-805.

8. Jiang M, Mishu MM, Lu D, Yin X. A case control study of risk factors and neonatal outcomes of preterm birth. Taiwan J Obstet Gynecol. 2018;57(6):814-8.

9. Oliveira LL, Gonçalves AC, Costa JS, Bonilha AL. Fatores maternos e neonatais relacionados à prematuridade. Rev Esc Enferm USP. 2016;50(3):382-9.

10. Rades É, Bittar RE, Zugaib M. Determinantes diretos do parto prematuro eletivo e oS resultados neonatais. Rev Bras Ginecol Obstet. 2004; 26(8):655-62.

11. Oliveira CS, Casagrande GA, Grecco LC, Golin MO. Perfil de recém-nascidos pré-termo internados na unidade de terapia intensiva de hospital de alta complexidade. ABCS Health Sci. 2015;40(1):28-32.

12. Gebreheat G, Tsegay T, Kiros D, Teame H, Etsay N, Welu G, et al. Prevalence and Associated Factors of Perinatal Asphyxia among Neonates in General Hospitals of Tigray, Ethiopia, 2018. Biomed Res Int. 2018;2018:5351010.

13. Noronha GA, Torres TG, Kale PL. Análise da sobrevida infantil segundo características maternas, da gestação, do parto e do recém-nascido na coorte de nascimento de 2005 no Município do Rio de Janeiro-RJ, Brasil. Epidemiol Serv Saúde. 2012;21(3):419-30.

14. Vanin LK, Zatti H, Soncini T, Nunes RD, Siqueira LB. Fatores de risco materno-fetais associados à prematuridade tardia. Rev Paul Pediatr. 2020; 38:e2018136.

15. Mwamakamba LW, Zucchi P. Estimativa de custo de permanência hospitalar para recémnascidos prematuros de mães adolescentes em um hospital público brasileiro. Einstein (Pão Paulo). 2014;12(2):223-9.

16. Rego MA, França EB, Travassos AP, Barros FC. Avaliação do perfil de nascimentos e óbitos em hospital de referência. J Pediatr (Rio J). 2010 ;86(4):295-302.

17. Stoll BJ, Hansen NI, Bell EF, Shankaran S, Laptook AR, Walsh MC, et al. Neonatal outcomes of extremely preterm infants from the NICHD Neonatal Research Network. Pediatrics. 2010;126(3):443-56.

18. Bouzada MC, Reis ZS, Vieira MN, Corrêa LP, Oliveira GM, Penido MG, et al. Resposta aos procedimentos de reanimação neonatal no quinto minuto de vida em recém-nascidos Apgar $\leq 3$ no primeiro minuto. Rev Med Minas Gerais. 2018;28(Supl.6):e-S280608.

19. Perlman JM, Wyllie J, Kattwinkel J, Wyckoff MH, Aziz K, Guinsburg R, et al. Part 7: neonatal resuscitation: 2015 International Consensus on Cardiopulmonary Resuscitation and Emergency Cardiovascular Care Science With Treatment Recommendations. Pediatrics. 2015;136(Suppl 2):S120-66.

20. Almeida MF, Guinsburg R, Costa J0, Anchieta LM, Freire LM, Campos Júnior D. Non-urgent caesarean delivery increases the need for ventilation at birth in term newborn infants. Arch Dis Child Fetal Neonatal Ed. 2010;95(5):F326-F30. 